\title{
La phase-3 du projet HOMONIM : définition et contenu
}

\section{Didier JOURDAN ${ }^{\mathbf{1}}$, Denis PARADIS ${ }^{\mathbf{2}}$, Audrey PASQUET ${ }^{\mathbf{1}}$, Héloïse MICHAUD ${ }^{\mathbf{1}}$, Rémy BARAILLE ${ }^{1}$, Laurie BISCARA ${ }^{1}$, Alice DALPHINET ${ }^{2}$, Patrick OHL ${ }^{2}$}

1. Shom,

Shom-Toulouse, 42 avenue Gaspard Coriolis, 31057 Toulouse, France.

didier.jourdan@shom.fr

2. Météo-France,

42 avenue Gaspard Coriolis, 31057 Toulouse, France.

denis.paradis@meteo.fr

\section{Résumé :}

Le projet HOMONIM - Historique, Observation, MOdélisation des NIveaux Marins - est un projet commun du SHOM et de Météo-France, sous maîtrise d'ouvrage de la Direction Générale pour la Prévention des Risques (DGPR).

Il s'attache depuis plusieurs années à développer une capacité de prévision multi-échelle des surcotes et des vagues jusqu'à la côte, à l'état de l'art, compatible avec les contraintes de mise en œuvre opérationnelle, et avec pour applications principales d'alimenter le dispositif de vigilance vagues-submersion de Météo-France et de mieux anticiper le risque de submersion marine.

La phase-3 du projet, qui va débuter au premier semestre 2020, a pour objectif :

- de contribuer à enrichir le patrimoine de données de référence existant (données bathymétriques, forçage météorologiques,...),

- d'améliorer le réalisme des modèles opérationnels à proximité immédiate de la côte,

- de fournir un contexte de modélisation très haute résolution, de référence, adapté à l'alimentation et l'interfaçage d'autres capacités de modélisation locale, des modèles de débordement ou d'outils empirique d'estimation d'impact à terre,

- de mieux appréhender les incertitudes sur les prévisions liées aux forçages atmosphériques et aux modèles mis en œuvre,

- de préparer la modélisation fine des processus littoraux pour un usage opérationnel ultérieur, tout en maîtrisant les enjeux de production opérationnelle temps réel en termes de temps calcul, de robustesse et de volumes de données générés par les chaînes opérées.

Mots-clés :

Submersions marines, Surcote, Vagues, Prévision, Vigilance, Opérationnalité 


\section{Thème 7 - Risques côtiers}

\section{Introduction}

Le projet Homonim, initié en 2011, est construit comme une contribution directe au plan d'action de la stratégie nationale de gestion des risques d'inondations pour : "augmenter la sécurité des populations exposées en développant la prévision", au travers de l'amélioration continue des capacités de modélisation qui alimentent le dispositif de la vigilance vagues-submersion (VVS) de Météo-France.

En phase 1 (2012-2015), le projet a ciblé les efforts sur la métropole. Il a conforté les outils de prévision de la VVS en réalisant l'extension du réseau d'observation des niveaux marins (six nouveaux marégraphes) et la refonte des chaînes opérationnelles de modélisation des surcotes et des vagues en zone côtière. Sur un plan plus prospectif, il a posé les bases scientifiques et technologiques de la modélisation pour prévoir les conditions météo-océaniques côtières à une échelle infra-départementale. Enfin, il a élargi la mise à disposition des résultats des modèles vers les utilisateurs spécialisés et le grand public via la mise en place de fonctionnalités spécifiques sur les différents portails de diffusion de Météo-France (http://donneespubliques.meteofrance.fr) et du Shom (http://data.shom.fr);

La phase-2 (2016-2019) a étendu ces réalisations pour doter les départements d'outremer de capacités de prévision similaires à celle en métropole, tout en continuant à faire progresser l'ensemble des systèmes de prévision et de diffusion déjà en place (dont l'introduction d'une prévision d'ensemble de surcotes).

La phase-3 du projet, qui va débuter au premier semestre 2020, vise à poursuivre l'amélioration des outils de prévision de l'aléa et à préparer la transition vers la prévision opérationnelle des débordements.

Après un rapide bilan des capacités de prévisions opérationnelles actuelles, nous décrivons le programme de travail de cette phase-3 du projet.

\section{Bilan des capacités opérationnelles de prévision}

\subsection{Les modèles et configurations}

Les configurations développées dans le projet s'appuient sur le modèle de surcote shallow water HYCOM, utilisé en mode barotrope (BARAILLE \& FILATOFF, 1995). et sur le modèle à phase moyennée de prévision des états de mer Wavewatch III (TOLMAN \& the WAVEWATCH III Development Group, 2014).

Les caractéristiques détaillées des configurations sont fournies dans les tableaux 1 et 2.

\subsection{Les scénarios opérationnels}

Les modèles sont opérés plusieurs fois par jour avec différents forçages météorologiques afin de permettre aux prévisionnistes d'évaluer les écarts induits sur les valeurs prévues des vagues, des surcotes et des hauteurs d'eau. L'échéance maximale de prévision est 


\section{XVIèmes Journées Nationales Génie Côtier - Génie Civil \\ Le Havre 2020}

variable selon le forçage (généralement $48 \mathrm{~h}$ pour les modèles Arome, et jusqu'à $120 \mathrm{~h}$ pour le modèle du centre européen IFS).

Tableau 1. Caractéristiques des configurations opérationnelles du projet Homonim.

\begin{tabular}{|c|c|c|}
\hline $\begin{array}{l}\text { Configuration / } \\
\text { Modèle }\end{array}$ & Vagues (WW3) & Surcotes (Hycom) \\
\hline Métropole ATL & $\begin{array}{l}\text { * Grille non structurée } \\
\text { * Résolution : } 200 \text { à 400m à la côte } \\
\text { * Carte de sédimentologie variable } \\
\text { * Forçage en courants et hauteurs } \\
\text { issus d'Hycom }\end{array}$ & $\begin{array}{l}\text { * Grille curvilinéaire } \\
\text { * Domaine : }-10^{\circ} \mathrm{E} / 9^{\circ} \mathrm{E} ; 43^{\circ} \mathrm{N} / 62^{\circ} \mathrm{N} \\
\text { * Résolution côtière : } 500 \mathrm{~m} \text { à } 1 \mathrm{~km} \\
\text { * Forçages de marée : atlas } \mathrm{NEA} 2011 \\
\text { * Friction de fond spatialement variable } \\
\text { et optimisée } \\
\text { * Coefficient de frottement par le vent } \\
\text { selon Charnock, } \alpha c=0,025\end{array}$ \\
\hline Métropole MED & $\begin{array}{l}\text { * Grille non structurée } \\
\text { * Résolution : } 200 \text { à } 400 m \text { à la côte } \\
\text { * Carte de sédimentologie variable } \\
\text { * Pas de forçage en courants et } \\
\text { hauteurs (faible marnage) }\end{array}$ & $\begin{array}{l}\text { * Grille curvilinéaire } \\
\text { * Domaine : }-9^{\circ} \mathrm{E} / 36^{\circ} \mathrm{E} ; 30^{\circ} \mathrm{N} / 45^{\circ} \mathrm{N} \\
\text { * Résolution côtière : } 1,5 \mathrm{~km} \\
\text { * Pas de forçage de marée (interactions } \\
\text { marée/surcote négligeables) } \\
\text { * Friction de fond quadratique constante } \\
\text { * Coefficient de frottement par le vent } \\
\text { selon Charnock, } \alpha c=0,025\end{array}$ \\
\hline Outre-Mer Indien & & $\begin{array}{l}\text { * Grille régulière } \\
\text { * Domaine : } 32^{\circ} \mathrm{E} / 67.5^{\circ} \mathrm{E} ; 8^{\circ} \mathrm{S} / 30^{\circ} \mathrm{S} \\
\text { * Résolution : 3,2km } \\
\text { * Forçages de marée : Atlas FES } 2014 \\
\text { * Friction de fond spatialement variable } \\
\text { optimisée } \\
\text { * Coef. de frottement par le vent, POND } \\
\text { \& PICKARD, } 1993, \text { (coupure à } 50 \mathrm{~m} / \mathrm{s} \text { ) }\end{array}$ \\
\hline Mayotte & $\begin{array}{l}\text { * Grille non structurée } \\
\text { * Résolution : } \\
\quad \text { 100m sur le lagon mahorais } \\
500 m \text { sur les Comores } \\
\text { * Carte de sédimentologie variable } \\
\text { * Forçage en courants et hauteurs } \\
\text { issus d'Hycom }\end{array}$ & $\begin{array}{l}\underline{\text { Zoom } 1} \\
* 42.5^{\circ} \mathrm{E} / 46.95^{\circ} \mathrm{E} ; 10.8^{\circ} / 13.83^{\circ} \mathrm{S} \\
* \text { Résolution côtière }: 800 \mathrm{~m} \\
\underline{\text { Zoom } 2} \\
* 44.65^{\circ} \mathrm{E} / 45.67^{\circ} \mathrm{E} ; 12.28^{\circ} \mathrm{S} / 13.41^{\circ} \mathrm{S} \\
* \text { Résolution côtière }: 200 \mathrm{~m} \\
\text { Zooms } 1 \text { et } 2^{*} \text { Friction de fond } \\
\text { spatialement variable optimisée } \\
\text { * Coef. de frottement par le vent, POND } \\
\text { \& PICKARD, 1993, (coupure à } 50 \mathrm{~m} / \mathrm{s} \text { ) }\end{array}$ \\
\hline
\end{tabular}




\section{Thème 7 - Risques côtiers}

\begin{tabular}{|c|c|c|}
\hline $\begin{array}{l}\text { Configuration / } \\
\text { Modèle }\end{array}$ & Vagues (WW3) & Surcotes (Hycom) \\
\hline La Réunion & $\begin{array}{l}\text { * Grille non structurée } \\
\text { * Résolution : } \\
200 m \text { sur La Réunion } \\
500 \text { m sur Maurice } \\
\text { * Forçage en courants et hauteurs } \\
\text { issus d'Hycom }\end{array}$ & $\begin{array}{l}\text { Zoom } 1 \\
* 53.6^{\circ} \mathrm{E}: 60.56^{\circ} \mathrm{E} ; 18^{\circ} \mathrm{S}: 22.58^{\circ} \mathrm{S} \\
\text { * Résolution côtière : } 800 \mathrm{~m} \\
\text { * Friction de fond spatialement variable, } \\
\text { optimisée } \\
\text { * Coef. de frottement par le vent, } P O N D \\
\text { \& PICKARD, } 1993, \text { (coupure à } 50 \mathrm{~m} / \mathrm{s} \text { ) }\end{array}$ \\
\hline Outre-Mer Caraïb & & \\
\hline Guyane & $\begin{array}{l}\text { * Grille non structurée } \\
\text { * Résolution : 200m à la côte } \\
\text { * Forçage en courants et hauteurs } \\
\text { issus d'Hycom }\end{array}$ & $\begin{array}{l}\text { * Grille curvilinéaire } \\
\left.\text { * Domaine : } 42^{\circ} \mathrm{W}: 64^{\circ} \mathrm{W} ; 0^{\circ} \mathrm{N}: 20^{\circ} \mathrm{N}\right] \\
\text { * Résolution côtière : } \\
\text { 900m sur les Antilles, } \\
2,3 \mathrm{~km} \text { sur la Guyane }\end{array}$ \\
\hline Antilles & $\begin{array}{l}\text { * Grille non structurée } \\
\text { * Résolution : 200m à la côte } \\
\text { * Forçage en courants et hauteurs } \\
\text { issus d'Hycom }\end{array}$ & $\begin{array}{l}\text { * Forçages de marée : Atlas FES } 2014 \\
\text { * Friction de fond spatialement variable } \\
\text { optimisée } \\
\text { * Coef. de frottement par le vent, POND } \\
\& \text { PICKARD, } 1993 \text {, (coupure à } 50 \mathrm{~m} / \mathrm{s} \text { ) }\end{array}$ \\
\hline
\end{tabular}

Tableau 2. Caractéristiques des forçages météorologiques des modèles de surcotes et de vagues.

\begin{tabular}{|c|c|c|c|c|}
\hline \multirow{2}{*}{ Modèle atmosphérique } & \multirow{2}{*}{$\begin{array}{l}\text { Nbre de run/jour } \\
\text { (réseaux de production) }\end{array}$} & \multirow{2}{*}{ Description } & \multicolumn{2}{|c|}{ Résolution } \\
\hline & & & Spatiale & temporelle \\
\hline \multirow[t]{2}{*}{ IFS (ECMWF) } & 4 & Spectral global $15 \mathrm{~km}$ & $0,125^{\circ}$ & 1 heure \\
\hline & $(0,6,12,18 U T C)$ & & & \\
\hline \multirow[t]{2}{*}{ Arpege (Météo-France) } & 4 & Spectral global $10 \mathrm{~km}$ & $0,1^{\circ}$ & 1 heure \\
\hline & $(0,6,12,18 U T C)$ & sur la France & & \\
\hline \multirow[t]{4}{*}{ Arome(Météo-France) } & 5 & Aire limitée couplé avec & $0,01^{\circ}$ & 1 heure \\
\hline & $(0,3,6,12,18 U T C)$ & modèle Arpege & & \\
\hline & & (1,3 km en métropole & & \\
\hline & & 2,5 km en outre-mer) & & \\
\hline \multirow[t]{2}{*}{ Arome-IFS (Météo-France) } & 2 & Aire limitée couplé avec & $0,025^{\circ}$ & 1 heure \\
\hline & $(0,12$ UTC $)$ & modèle IFS (2,5 km) & & \\
\hline
\end{tabular}




\section{XVIèmes Journées Nationales Génie Côtier - Génie Civil \\ Le Havre 2020}

\subsection{Apports et limitations des systèmes actuels}

Ces capacités fournissent une information brute à destination des responsables d'alerte et de gestion de crise sur l'intensité et la dangerosité d'une situation météo-océanographique et contribuent, après expertise des prévisionnistes, au dispositif Vigilance Vagues Submersion, à l'échelle du département.

En l'état actuel, ces capacités de prévision présentent encore des limitations au regard des besoins de :

- connaitre l'aléa plus finement le long de la côte ;

- connaitre les conséquences à terre ;

- pré-positionner ou déployer les moyens de façon différenciée sur une partie du territoire plutôt qu'une autre ;

- décider des fermetures plus ciblées de routes, d'accès, d'infrastructures...;

- décider d'éventuelles évacuations les plus ciblées possible.

Ces limites relèvent de quelques points majeurs à améliorer :

- le manque de résolution des grilles ;

- le formalisme limitant des modèles utilisés (modèle de circulation hydrodynamique 2D hydrostatique et modèle d'état de mer spectral) pour décrire la dynamique et les processus de très haute résolution (THR - résolution de quelques dizaines de mètres), voire de extra haute résolution (XTHR - résolution de quelques mètres) qui se développent dans la bande littorale ;

- le manque d'observations sur de longues périodes et d'événements majeurs à rejouer, en particulier sur l'outre-mer, pour encore mieux contrôler et affiner les paramétrisations.

\section{Description du contenu de la phase-3.}

Deux axes de développement ont été retenus pour repousser ces limitations :

- la montée en résolution spatiale par l'adaptation des modèles de surcotes aux grilles non-structurées ;

- l'amélioration de la physique des modèles par:

o la prise en compte de l'effet des vagues sur le niveau moyen près de la côte (wavesetup) ;

o la prise en compte des effets non-hydrostatiques pour une modélisation "unique" XTHR des vagues, surcotes et niveaux.

En plus de ces objectifs, le projet se doit aussi de maitriser un certain nombre d'enjeux opérationnels sans lesquels une production et une exploitation temps réel pour l'aide à la gestion de crise est impossible :

- le coût en temps de calcul des prévisions : l'exploitation en temps réel des résultats par les prévisionnistes implique une optimisation des codes à l'infrastructure utilisée et une bonne "scalabilité" des codes parallélisés. En outre, l'utilisation d'une prévision d'ensemble exige d'avoir des déclinaisons peu coûteuses des modèles déterministes ; 


\section{Thème 7 - Risques côtiers}

- le volume de données : le coût calcul du post-traitement des résultats des modèles et le transfert sur les réseaux imposent de maîtriser le volume de ces données ;

- la robustesse des chaînes opérationnelles opérées : la robustesse attendue impose de simplifier les chaînes au maximum, en limitant le nombre de configurations (régions), en allégeant le flux des données et en restreignant la complexité des systèmes couplés et les emboîtements de grilles. Elle implique aussi une robustesse des codes numériques.

Le projet est organisé selon 6 axes de travail.

\subsection{Axe 1: Maintien en conditions opérationnelles et à l'état de l'art des systèmes en $\underline{\text { service }}$}

Cet axe permet de faire face aux évolutions des moyens de calcul haute performance, des modèles atmosphériques, des besoins des usagers et aux opportunités d'amélioration des codes actuels.

Il couvre les travaux :

- d'adaptation des codes et paramétrisations des configurations actuelles aux mises à jour scientifiques communautaires et de l'équipe projet ;

- d'adaptation des chaînes opérationnelles aux évolutions des codes et de l'environnement de calcul ;

- d'adaptation des chaînes aux évolutions des modèles atmosphériques ;

- d'adaptation de la production opérationnelle pour satisfaire les demandes utilisateurs.

\subsection{Axe 2: Acquisition des données de référence complémentaires}

Cet axe comprend les tâches :

- d'organisation et de réalisation d'une campagne de mesure dédiée, indispensable à la validation des travaux sur le non-hydrostatisme et les processus littoraux (axe 4);

- de production de MNT spécifiques pour les axes de modélisation (axes 3 et 4);

- d'expertise, sélection et bancarisation de nouveaux évènements atmosphériques pour permettre les évolutions des configurations des modèles de vagues et de surcotes.

\subsection{Axe 3: Amélioration des systèmes de prévision Haute Résolution (HR) et Très}

Haute Résolution (THR) couplé

Cet axe consiste, en premier lieu, à adapter le code de prévision des surcotes aux grilles non structurées, afin d'augmenter et d'optimiser localement la résolution jusqu'à la centaine de mètres où c'est nécessaire. Le but est de représenter la complexité de la dynamique océanique côtière, tout en diminuant les temps de calcul et la complexité de la chaîne de production.

L'option retenue est une refonte du modèle HYCOM actuel en un modèle TOLOSA sur la base d'un code numérique développé comme une boîte à outils permettant de faciliter la mise en œuvre d'un modèle numérique sur un maillage non structuré. 


\section{XVI'èmes Journées Nationales Génie Côtier - Génie Civil \\ Le Havre 2020}

Ce choix est porté par :

- le fort historique scientifique sur le code numérique, qui s'appuie les travaux de l'ONERA et l'Institut de Mécanique de Toulouse (GRENIER et al., 2011 et 2013);

- la pérennisation de l'expérience et des performances acquises dans les précédentes phases, via le transfert dans TOLOSA du schéma de discrétisation des équations utilisé dans HYCOM ;

- un environnement assurant la reproductibilité des résultats du système actuel dans la mesure où TOLOSA permet de gérer des maillages structurés et des grilles duales.

Les travaux comprennent :

- le développement numérique du modèle de surcotes TOLOSA sur grille non structurée et son adaptation à la modélisation océanographique (niveaux et courants) ;

- le développement d'une configuration de prévisions des surcotes, HR, régionale Atlantique non structurée (TOLOSA_ATL);

- le développement d'une configuration TOLOSA de prévisions des surcotes, THR, Nord-Aquitaine (du bassin d'Arcachon à l'île Ré) ;

- le développement d'une configuration WW3 de prévisions des vagues, THR, NordAquitaine ;

- le développement du système couplé, THR, Nord-Aquitaine (TOLOSA/WW3_NAQ). Après validation et vérification de la non régression des performances, les configurations TOLOSA_ATL et TOLOSA/WW3_NAQ donneront lieu à deux nouveaux systèmes opérationnels mis en service et opérés à Météo-France :

- un système de prévision des surcotes HR, d'emprise identique à celle en service avec des résolutions atteignant $200 \mathrm{~m}$ de résolution à la côte permettant :

o d'affiner géographiquement les prévisions opérationnelles sur toutes les façades pour une analyse plus sectorisée des conditions météo-océanographiques sur le littoral ;

o de permettre l'implémentation généralisée de la modélisation couplée THR.

- un système de prévision THR couplé surcote/vagues sur la zone Nord-Aquitaine permettant :

o d'améliorer la précision des hauteurs d'eau prévues (prise en compte du wave setup) sur des zones à enjeux et complexes (Bassin d'Arcachon et pertuis charentais) ;

o de fournir des prévisions numériques brutes adaptées à l'exploitation pour la VVS infra-départementale ;

o d'étudier l'interfaçage entre des systèmes THR opérationnels et XTHR locaux (axe 4).

En parallèle du développement des configurations régionale HR et de façade THR, une tâche est prévue pour étudier la capacité du modèle TOLOSA non structuré à reproduire la dynamique en zone estuarienne. Pour cela :

- une configuration du modèle sera développée sur l'estuaire de la Gironde ; 


\section{Thème 7 - Risques côtiers}

- les résultats du modèle seront confrontés aux résultats des modèles dédiés comme TUGO pour la marée ou Telemac pour les niveaux d'eau sur la Gironde, jusqu'en limite amont du régime maritime.

\subsection{Axe 4 : Modélisation eXTra Haute résolution des processus littoraux}

C'est un axe préparatoire pour l'avenir. Il s'attache à acquérir, développer et implémenter la modélisation non hydrostatique dans le modèle TOLOSA, étape indispensable pour envisager de faire, ou d'alimenter correctement, des modèles dynamiques de débordement.

C'est une macro-tâche qui inclut :

- l'étude et l'ajout des équations non hydrostatiques dans le modèle TOLOSA (TOLOSA-NH) ;

- l'évaluation des performances TOLOSA-NH sur les processus littoraux au travers :

o de cas tests académiques (confrontation aux données de laboratoire ou aux résultats de modèles de référence comme Symphonie);

o du développement d'une maquette de prévision XTHR sur une zone restreinte (quelques $\mathrm{km}^{2}$ ) au nord de l'île de Ré ;

o de la validation de la maquette dans les conditions très littorales en utilisant les données de la campagne (axe 2).

\subsection{Axe 5 : Prévision d'ensemble}

Les tâches de cet axe incluent :

- l'évaluation du coût calcul de plusieurs configurations de PE de vagues en côtiers, en modulant la résolution spatiale et le modèle d'ensemble atmosphérique choisi, et l'étude de la pertinence des PE de vagues obtenues selon ces différentes configurations ;

- l'étude de la pertinence d'inclure une double paramétrisation de la physique des vagues dans l'ensemble de vagues ;

- la mise en opérationnel de la configuration de PE de vagues en côtier retenue ;

- le développement, avec l'aide des prévisionnistes, du post-traitement des sorties brutes des modèles d'ensemble (vagues et surcotes) à réaliser pour optimiser leur exploitation lors de l'élaboration de la VVS.

\subsection{Axe 6: Soutien à la valorisation de la donnée au profit de la gestion de crise}

Cet axe regroupe les tâches permettant de s'articuler et d'interagir avec les gestionnaires de crise, avec les autres acteurs de la modélisation (privés ou institutionnels) et de faire du retour d'expérience. Il a aussi pour objectif de rendre visible les produits et services qui résultent des productions opérationnelles.

A ce titre cet axe inclut: 


\section{XVIèmes Journées Nationales Génie Côtier - Génie Civil \\ Le Havre 2020}

- une activité de définition du concept d'emploi des nouveaux produits et services pour la mise en œuvre de la VVS : raffinement des tableaux de seuils, utilisation de la modélisation couplée à THR ;

- l'accompagnement de la mise en place de la mission Référent Départemental Inondation ;

- le maintien en conditions des capacités de diffusion Shom (http://data.shom.fr) et Météo-France (http://donneespubliques.meteofrance.fr);

- l'organisation d'une journée technique nationale pour communiquer sur les travaux du projet auprès des utilisateurs potentiels.

\section{Références}

BARAILLE R., FILATOFF N. (1995). Modèle shallow-water multicouches isopycnal de Miami. Rapport technique. 003/95, SHOM.

TOLMAN H. L. and the WAVEWATCH III ${ }^{\circledR}$ Development Group (2014). User manual and system documentation of WAVEWATCH III version 4.18. Technical report.

GRENIER N., VILLEDIEU P., VILA J.-P. (2011). Schéma bas-Mach précis pour des écoulements à interface. 20ème Congrès Français de Mécanique, Besançon, 29 août au 2 septembre 2011, Ed. Association Française de Mécanique. http://hdl.handle.net $/ 2042 / 46342$

GRENIER N., VILA J.-P., VILLEDIEU P. (2013). An accurate low-Mach scheme for a compressible two-fluid model applied to free-surface flows. Journal of Computational Physics, Vol. 252, pp 1-19. https://doi.org/10.1016/j.jpp.2013.06.008

POND S., PICKARD G.L. (1993). Introductory dynamical oceanography, Ed. Elsevier Butterworth-Heinemann, $349 \mathrm{p}$. 
Thème 7 - Risques côtiers 